\title{
Evolution des lignées pseudocompatibles de betterave fourragère et sucrière (Beta vulgaris L.) au cours des générations d'autofécondation
}

\author{
F. Le Cochec et P. Soreau \\ avec la collaboration technique de J.M. Retailleau \\ INRA, station d'amélioration des plantes, Domaine de la Motte au Vicomte B.P. 29, 35650 Le Rheu, France
}

(reçu le 16 janvier 1989, accepté le 23 septembre 1989)

\begin{abstract}
Résumé - Un grand nombre de lignées pseudocompatibles de betterave fourragère et sucrière ont été autofécondées de 1983 à 1986 en utilisant des isoloirs de $75 \mathrm{dm}^{3}$. Entre les générations $I_{1}$ à $I_{7}$ le poids moyen de glomérules récoltés dans chaque isoloir croît régulièrement de 3,79 à $11,87 \mathrm{~g}$ et la proportion de glomérules viables de 30,32 à $63,67 \%$. Les valeurs médianes de chaque distribution suivent une progression analogue, respectivement de 2,54 à $9,20 \mathrm{~g}$ et de 19,44 à $68,41 \%$. Cette évolution est la même chaque année, qu'il s'agisse de betterave fourragère ou sucrière.

L'autofécondation est un moyen permettant de sélectionner des lignées de betterave de plus en plus pseudocompatibles. Les avantages et les inconvénients de cette méthode sont discutés. En moyenne, il est possible d'obtenir des lignées pseudocompatibles à partir de $46,5 \%$ des plantes $I_{0}$ sélectionnées dans une population d'origine. Le pourcentage d'allofécondation des lignées obtenues est variable suivant les lignées et il ne dépend pas de leur degré de consanguinité. Le déterminisme génétique de la pseudocompatibilité est distinct du système génétique qui contrôle l'incompatibilité chez la betterave.
\end{abstract}

betterave - pseudocompatibilité - incompatibilité - autofécondation

Summary - Evolution of pseudocompatible fodder and sugar beet lines (Beta vulgaris L.) during selfing generations. Numerous pseudocompatible fodder and sugar beet lines were selfed between 1983 and 1986 using 75 litre isolators (Figs 1, 2). From $S_{1}$ to $S_{7}$ generations, the harvested cluster mean weight inside each isolator regularly increased from 3.79 to 11.87 grams and the viable cluster percentage from 30.32 to 63.67 . For each distribution, median values followed the same progression : from 2.54 to 9.20 grams and from 19.44 to $68.41 \%$. This evolution is the same each year for both fodder and sugar beet lines (Tables I, II, III). If we suppose that sugar beet is issued by man selection from white fodder beet roots, the pseudocompatibility system remained unchanged during this long time procedure. Selfing is a mean of permitting the selection of increasingly pseudocompatible beet lines and the advantages and disadvantages of this method are discussed. On average, $46.5 \%$ of the $S_{0}$ originally selfed plants give rise to PSC lines. The outcrossing percentage of PSC lines is variable and non-correlated with their inbreeding level. The genetic background of pseudocompatibility in beet is unrelated to the incompatibility genetic system.

fodder and sugar beet - pseudocompatibility - incompatibility - inbreeding

\section{INTRODUCTION}

Les variétés actuelles de betterave sucrière ou fourragère sont caractérisées à la fois par une productivité et une qualité industrielle ou fourragère élevées et par une bonne adaptation à des conditions de milieu et de culture très différentes. Cependant, en raison de l'extrême complexité de leur constitution génétique (Hilleshog, 1974), elles présentent plusieurs inconvénients : il est très difficile de les décrire, de les distinguer et donc de les reconnaître et de certifier leur origine. Pour la même raison, on peut mettre en doute la stabilité de leur composition génétique au cours des années. La conséquence, peut-être la plus grave, de cette situation est que ces variétés ne permettent pas une mesure précise du progrès génétique.

Il a été proposé (Le Cochec, 1982) de réduire ces inconvénients en remplaçant, dans les formules hybrides, les familles hétérozygotes par des lignées fixées et bien définies. Ces lignées 
peuvent être obtenues par autofécondations répétées à partir de populations de base améliorées par sélection récurrente, à la fois pour leur valeur propre et pour leur valeur en combinaison. Au bout de 3 ou 4 générations, la plupart d'entre elles s'autofécondent sans difficultés particulières en conditions contrôlées. II faut bien voir cependant qu'il ne s'agit pas de véritables lignées autocompatibles, car elles peuvent accepter plus ou moins facilement le pollen étranger. Elles sont, de ce fait, considérées comme des lignées pseudocompatibles. La variété de betterave fourragère Trestel obtenue par cette méthode a été inscrite et commercialisée depuis 1975 dans plusieurs pays de la CEE. Elle se distingue nettement des autres variétés de betterave par son homogénéité et sa stabilité.

La betterave est une plante allogame, anémophile, considérée comme auto-incompatible avec un système d'incompatibilité contrôlé par deux séries alléliques, S et Z (Owen 1942, Maletsky \& Weisman, 1978). D'après Larsen (1977a), ce mécanisme serait en réalité plus complexe et comporterait au moins 4 séries alléliques à action complémentaire. Ce système d'incompatibilité devrait permettre d'affirmer, sans ambiguïté possible, si un croisement ou une autofécondation en conditions contrôlées aboutit à une réaction de compatibilité ou d'incompatibilité. L'autofécondation d'une plante individuelle ne révèle que rarement une distinction aussi nette et on constate le plus souvent tous les intermédiaires entre l'autocompatibilité et l'auto-incompatibilité complètes.

Cette fertilité partielle ou pseudocompatibilité correspondrait à une modification du système d'auto-incompatibilité ou plus exactement à la révélation, par une pression de sélection appropriée, d'une possibilité d'autofécondation dans une espèce naturellement auto-incompatible (Dana \& Ascher, 1985). La pseudocompatibilité se caractérise par une grande variabilité dans les quantités de graines obtenues par autofécondation et par une grande sensibilité aux conditions du milieu réalisé à l'intérieur de l'isoloir. En l'absence d'allopollen, l'autopollen peut germer sur les stigmates et se développer dans le style mais sa croissance est beaucoup plus lente que celle de l'allopollen et elle peut s'interrompre avant fécondation (Savitsky, 1950). En cela la pseudocompatibilité diffère nettement de l'autocompatibilité véritable conditionnée chez la betterave par la présence de l'allèle $S_{f}$ et qui se caractérise par une autofertilité complète et durable.

La pseudocompatibilité se rencontre dans plusieurs espèces allogames mais elle a été peu utilisée dans l'amélioration de ces espèces, sans doute parce qu'elle décroît avec le degré de consanguinité. Elle serait en relation avec une absence totale ou complète d'incompatibilité au niveau du style et résulterait de l'interaction de gènes modificateurs de l'activité des gènes de la série S (Henny \& Ascher, 1976).

Les premières études sur l'obtention de glomérules et de lignées de betterave par autofécondation datent du début du siècle (Shaw, 1916) et elles ont été poursuivies régulièrement depuis : Nilsson (1923), Vilmorin (1923), Grinko (1930), Stewart (1933), Brewbaker (1934), Kohls \& Down (1934), Archimovitsch (1948), Bandlow (1965), Oldemeyer \& Smith (1965), Codrescu (1974), Dobrosotvkov (1979), Vashchenko \& Yusubov (1986).

Ces auteurs ont comparé l'influence de différents milieux, de différents types d'isoloirs et de matériaux isolants (papier, cellophane, tissus divers) sur la production de glomérules en autofécondation et les lignées ont été suivies jusqu'à un stade plus ou moins avancé $\left(l_{1}\right.$ à $\left.I_{7}\right)$. Les résultats obtenus varient beaucoup suivant la méthode utilisée et suivant les conditions d'expérimentation, de sorte que les conclusions sont souvent limitées et contradictoires.

La pseudocompatibilité a été largement utilisée, depuis 1955, aux Etats-Unis par les compagnies de sélection privées pour l'obtention de lignées mâle-stériles monogermes et de lignées pollinisatrices de betterave à sucre (Oldemeyer, 1986). En URSS et au Japon, plusieurs auteurs ont obtenu des lignées de betterave par autofécondation mais l'importance réelle de ces lignées en sélection ne nous est pas connue. En Europe Occidentale, une partie des lignées monogermes mâle-stériles possèdent l'allèle d'autofertilité $S_{f}$ (cet allèle était lié au caractère monogerme dans les introductions provenant des Etats-Unis faites à partir de 1950) et la pseudocompatibilité commence seulement à être utilisée pour l'obtention de lignées pollinisatrices bien définies.

En raison de la fréquence très faible de l'allèle $S_{f}$ dans nos populations de base, la pseudocompatibilité a joué un rôle essentiel dans l'obtention de lignées fixées.

Le but de cet article est d'étudier, sur un grand nombre de lignées de betterave fourragère et sucrière, l'évolution de la pseudocompatibilité (poids et viabilité des glomérules) en fonction des générations d'autofécondation et d'essayer d'en préciser la nature et l'origine.

\section{MATÉRIEL ET MÉTHODES}

Les autofécondations ont été réalisées de 1983 à 1986 à la station d'amélioration des plantes, Le Rheu près de Rennes, sur des lignées $I_{1}$ à $I_{7}$ issues de 
populations de base diploïdes, auto-incompatibles et multigermes. Ces populations, très différenciées, ont été améliorées préalablement par sélection massale maternelle. Chaque année 15 à 30 racines $l_{0}$ par population ont été autofécondées dans 5 à 12 populations de base. Au total, au cours des 4 années, 16 populations de betterave fourragère (à racines jaunes, orange ou rouges et à teneur variable en matière sèche) et 23 populations de betterave sucrière de types très pauvres à très riches en sucre ont été utilisées comme source des nouvelles lignées.

A partir de la génération $I_{0}$ d'origine, les portegraines ont été conduits en génération annuelle (semés en châssis froids le 20 septembre, repiqués au printemps et récoltés entre le 25 et le 30 août de l'année suivante). Les autofécondations ont été poursuivies jusqu'à la $7^{e}$ génération. Pour chaque lignée, le passage d'une génération à la suivante a été réalisé en autofécondant 1 plante (exceptionnellement 2) choisie(s) au hasard dans cette lignée avant la floraison. Une sélection pour la valeur propre et la valeur en combinaison, entreprise à partir de la génération $l_{4}$, a entraîné l'élimination de nombreuses lignées au cours des générations suivantes. Cette réduction progressive du nombre des lignées suivies n'est pas en relation avec une diminution de la vigueur ou de la fertilité des porte-graines, qui sont relativement peu affectées par l'inbreeding, mais avec la sélection des caractères végétatifs dans les descendances.

Le type d'isoloir utilisé (Fig. 1) est constitué par une armature métallique de $0,40 \mathrm{~m}$ de diamètre et d'un volume total de $75 \mathrm{dm}^{3}$, disposée à l'extrémité d'un support métallique de $0,90 \mathrm{~m}$ de longueur, enfoncé de $0,40 \mathrm{~m}$ dans le sol. Le matériau d'isolement est une popeline de coton comportant $60 \mathrm{fils} / \mathrm{cm}$ dans un sens et 30 fils $/ \mathrm{cm}$ dans l'autre sens. L'examen de ce tissu au microscope indique qu'il permet de réaliser simultanément une protection à peu près complète contre le pollen extérieur et une bonne aération à l'intérieur de l'isoloir. Des expérimentations préalables ont indiqué qu'un volume d'isolement inférieur à $50 \mathrm{dm}^{3}$ ou un matériau d'isolement plus ou moins imperméable à l'air, créent des conditions d'éclairement, de température et d'humidité très défavorables à l'expression de la pseudocompatibilité.

Pour chaque plante, 3 à 4 j en moyenne avant l'ouverture des premières fleurs, 4 hampes florales sont enfermées dans l'isoloir dont l'étanchéité est assurée par un tampon de coton. Avant la fermeture de l'isoloir, le volume d'air emprisonné est traité abondamment à l'aide d'une solution aqueuse aphicide qui a la proprié-

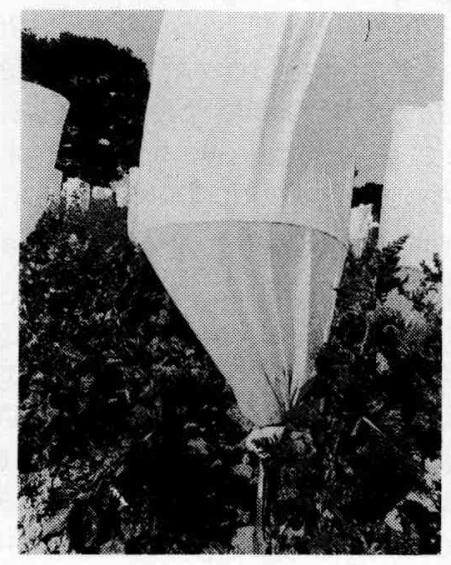

Fig. 1. Type d'isoloir utilisé. té de détruire le pollen ambiant ou présent sur la plante (Lynes \& Cormany, 1942). Scott (1970), indique en effet que les concentrations de pollen au-dessus d'un champ de porte-graines en floraison atteignent et dépassent fréquemment $50 \mathrm{grains} / \mathrm{dm}^{3}$ soit plus de 4000 grains par isoloir. D'après ces auteurs, le pollen peut rester viable à l'intérieur des isoloirs pendant 8 à 10 j ou même davantage.

Les hampes récoltées à maturité dans chaque isoloir sont séchées, battues et égrenées. Le poids des glomérules récoltés ( $\mathrm{gr}$ ) est estimé après nettoyage et tamisage au tamis $3 \mathrm{~mm}$. On constate chaque année à la récolte des différences importantes pour le taux de pseudocompatibilité entre les plantes d'une même génération (Fig. 2).

Le pourcentage de glomérules viables, (\% gv) c'est-à-dire de cymes* ayant au moins une graine fertile, est déterminé par le test dit du marteau. Pour cela, une vingtaine de glomérules de chaque lot sont écra-

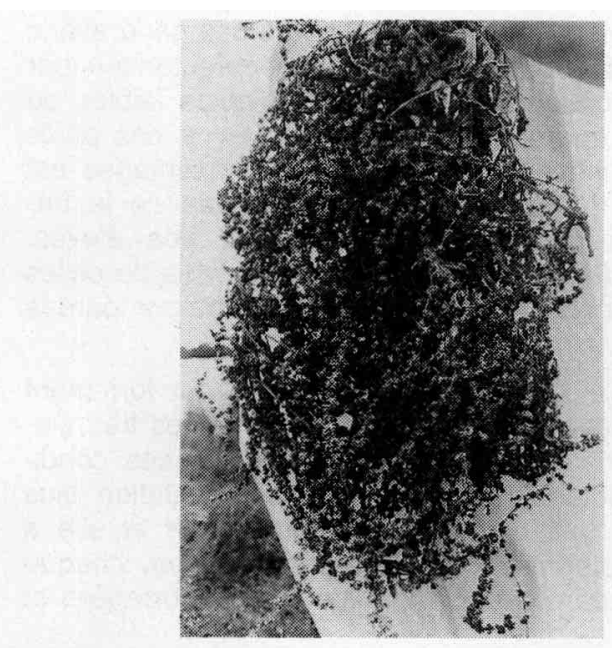

Fig. 2a. Pseudocompatibilité élevée ( $\left.l_{1} 1988\right)$.

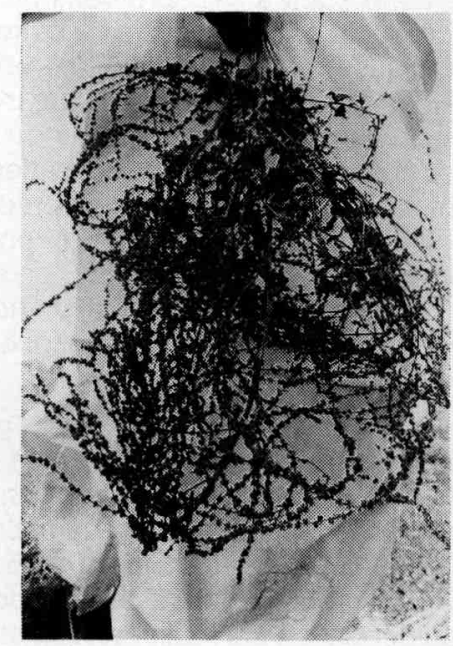

Fig. 2b. Pseudocompatibilité faible $\left(l_{1} 1988\right)$.

\footnotetext{
- Les fleurs de betterave sont groupées à l'aisselle d'une bractée en une inflorescence très courte, une cyme, qui donne naissance à maturité à un glomérule comportant 3 a 8 fruits contenant chacun une graine. Le nombre moyen de cymes par isoloir (dénombré sur 32 isoloirs) est en moyenne de $4450 \pm 674$
} 
sés sur une surface plane et dure. Les graines viables sont aisément identifiables par la présence des amandes. Longden et al. (1971) indiquent qu'il existe un coefficient de corrélation de 0,79 entre l'aspect des amandes déterminé par radiographie et la viabilité des graines estimée dans les essais officiels de germination. On peut donc, en première approximation, établir une relation entre l'aspect des amandes et leur viabilité. Ce test, rapide et facile à mettre en œuvre, a toutefois l'inconvénient d'entraîner la destruction des glomérules examinés.

Les moyennes des 2 caractères, poids des glomérules récoltés et pourcentage de glomérules viables, ont été calculées chaque année, pour chaque génération, puis séparément pour la betterave fourragère et sucrière.

Dans les premières générations les courbes de distribution des fréquences présentent un maximum pour les poids ou les pourcentages faibles ou nuls, une chute rapide et une fréquence réduite pour les poids ou les pourcentages élevés. Au cours des générations suivantes, la courbe des poids est caractérisée par une diminution de la fréquence des poids faibles ou nuls et une augmentation de la fréquence des poids moyens ou élevés. La courbe des pourcentages est caractérisée par un accroissement rapide de la fréquence des pourcentages élevés ou très élevés. Toutes ces distributions sont très différentes de celles d'une courbe normale et elles n'ont pu être comparées entre elles.

Pour les 2 caractères, la moyenne est fortement influencée par les poids ou les pourcentages très élevés caractérisant certaines plantes. Dans ces conditions, la médiane a semblé une représentation plus réaliste que la moyenne de la distribution et elle a donc été déterminée, à chaque génération, chaque année et séparément pour la betterave fourragère et sucrière.

Dans une étude de la pseudocompatibilité, il est important de vérifier au préalable que les valeurs déterminées à l'intérieur de l'isoloir ne sont pas différentes de celles existant à l'air libre dans un isolement par la distance. A cet effet, 4 racines de betterave fourragère et 4 racines de betterave sucrière ont été coupées en 2 parties égales en 1986. Chaque moitié a été autofécondée suivant la méthode décrite ou isolée par la distance. L'absence de pollen de betterave dans ces isolements avait été vérifiée l'année précédente en y cultivant une plante mâle-stérile. Le pourcentage moyen de cymes fécondées dans ces 8 endroits a été de $0,4 \pm 0,2 \%$ (entre 0,0 et $1,4 \%$ ) soit un nombre très réduit et on a donc estimé que l'atmosphère de ces lieux était exempte de pollen de betterave.

Un certain nombre de lignées sont perdues à chaque génération en raison du maintien d'un taux plus ou moins élevé de plantes ne pouvant s'autoféconder. On a calculé, pour chaque génération $I_{1}$ à $I_{7}$, le nombre des lignées ayant au moins $1,3,5,10$ ou 20 plantes, à partir du regroupement général des lignées. Un tel tableau permet en particulier d'estimer, pour une génération donnée, le pourcentage de plantes autofécondées en $l_{0}$ susceptibles de donner des descendances d'une importance définie au préalable.

Une lignée pseudocompatible peut également être caractérisée par son pourcentage d'allofécondation en présence de pollen étranger. Ce pourcentage a été calculé, chaque année, sur 3 lignées à hypocotyle vert (caractère récessif) choisies au hasard dans chaque génération, soit 60 lignées $l_{1}$ à $l_{7}$ au total, de 1983 à 1986. Une plante entière de chacune de ces lignées a été croisée dans un grand isoloir de popeline de coton $\left(1000 \mathrm{dm}^{3}\right.$ ) avec une plante de même précocité de floraison provenant d'une lignée fixée homozygote pour le caractère hypocotyle rouge (caractère dominant). Le pourcentage de plantes croisées a été déterminé l'année suivante sur un nombre compris entre 85 et 496 plantes par lignée.

Enfin, pour suivre d'une manière plus précise l'évolution des descendances d'une plante $I_{0}$ pour les 2 caractères étudiés, 10 plantes entières de la génération $l_{1}$ ont été autofécondées en 1985 sous un grand isoloir de $1000 \mathrm{dm}^{3}$ et ensuite une plante entière dans chaque descendance au cours des générations $I_{2}$ et $\mathrm{I}_{3}$.

\section{RÉSULTATS}

Le poids moyen et médian des glomérules récoltés ainsi que le pourcentage moyen et médian des glomérules viables d'une plante sous isoloir sont présentés pour les générations $I_{1}$ à $I_{7}$, d'une part pour les lignées de betterave sucrière, de betterave fourragère et l'ensemble des lignées (Tableau I) et d'autre part chaque année de 1983 à 1986 (Tableau II).

Pendant les 4 années, pour l'ensemble des lignées en cours de fixation, le poids moyen des glomérules récoltés par isoloir augmente régulièrement au cours des générations de 3,79 à $11,87 \mathrm{~g}$ (soit environ de 220 à 780 glomérules) et le pourcentage des glomérules viables de 30,32 à 63,67 .

La même évolution pour les 2 caractères est constatée dans l'analyse séparée du comportement des lignées de betterave fourragère et de betterave sucrière au cours des 4 années. Dans l'ensemble, les 2 caractères évoluent parallèlement dans les 2 types de plantes. Les variétés actuelles de betterave sucrière proviennent d'une sélection massale dans la variété Blanche de Silésie, obtenue elle-même au début du 190 siècle par Achard et Von Koppy à partir de variétés de betteraves fourragères blanches alors cultivées en Allemagne (Coons et al., 1955). Cette sélection n'a apparemment pas différencié le système d'incompatibilité propre à la betterave fourragère et à la betterave sucrière ni leur comportement pour la pseudocompatibilité.

Les résultats annuels font apparaître certaines différences entre années, notamment en raison des valeurs élevées obtenues dans la génération $\mathrm{I}_{1}$ en 1984 et des faibles valeurs de la génération $\mathrm{I}_{4}$ en 1985. II est difficile cependant d'affirmer que les années sont différentes pour les 2 caractères puisque ces différences ne se manifestent que sur une génération chaque année. On peut seulement remarquer que les températures 


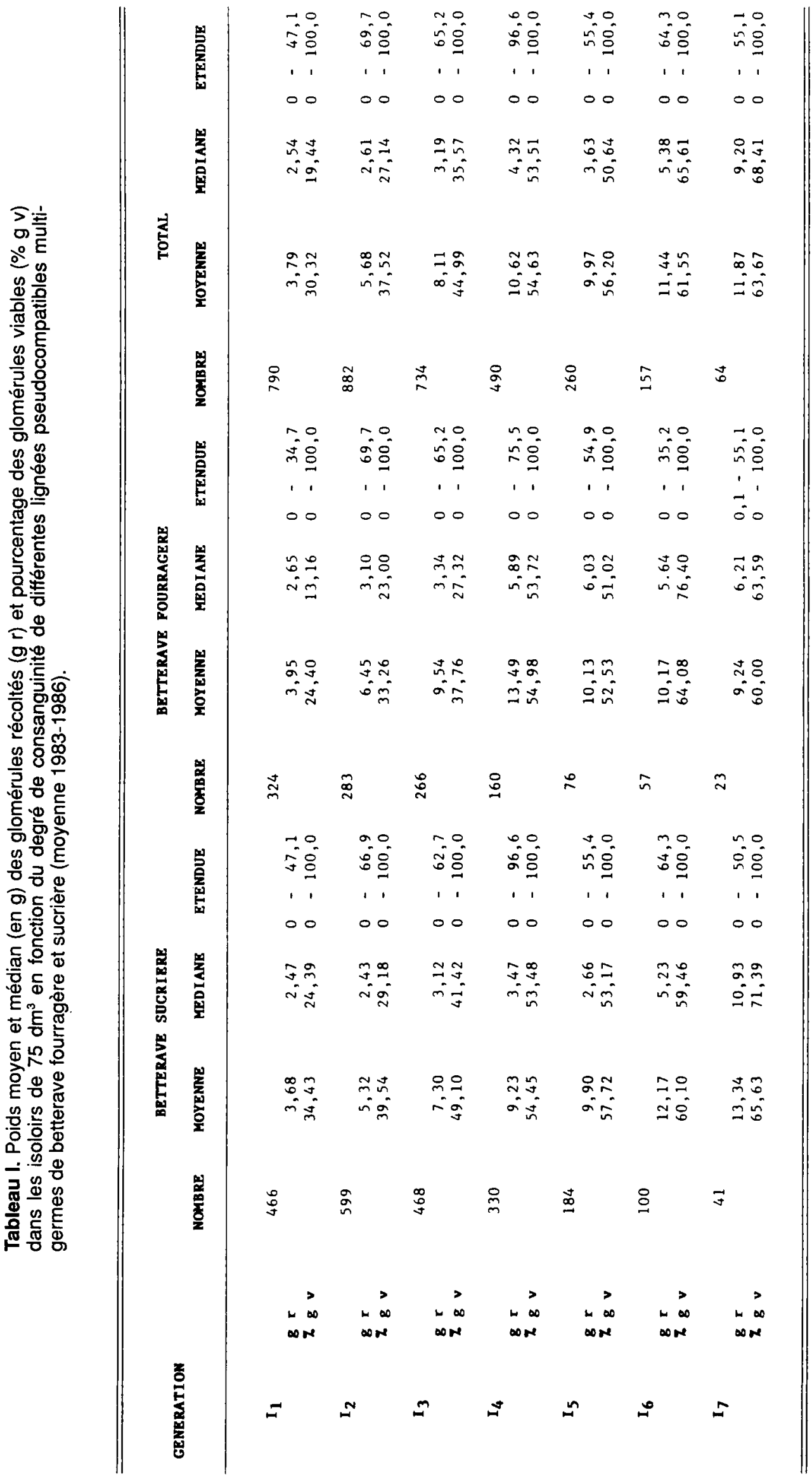




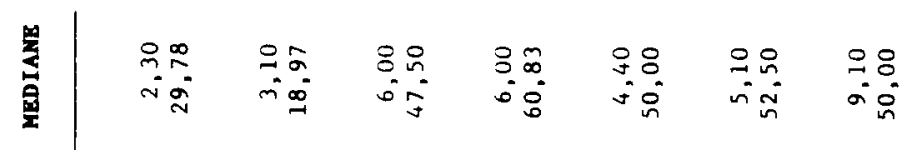

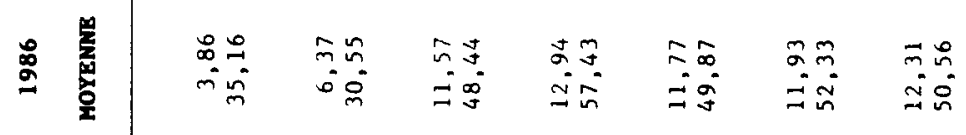

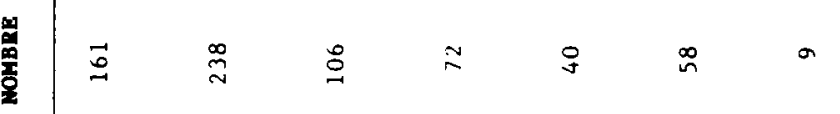

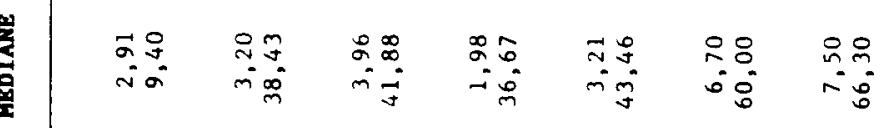

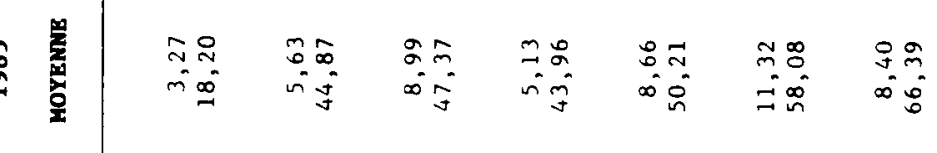

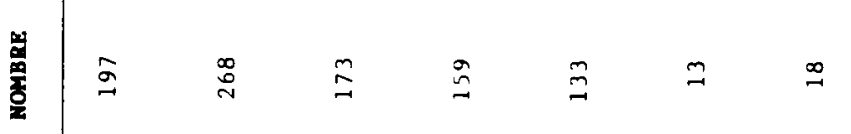

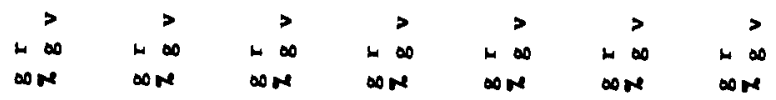


moyennes enregistrées en 1984 entre le 25 juin et le 20 juillet, époque correspondant à la fécondation et aux premières divisions de l'embryon, sont relativement basses (comprises entre 16,2 et $17,3^{\circ}$ ). Ces températures sont considérées par plusieurs auteurs (Oldemeyer \& Smith, 1965; Weisman, 1981) comme les plus favorables à l'expression de la pseudocompatibilité. Le Tableau II permet aussi de suivre la filiation ou l'évolution de la descendance d'un ensemble de lignées dans le temps : ainsi la descendance des lignées $I_{1}$ de 1983 jusqu'à la génération $I_{4}$ en 1986. On constate la même régularité d'accroissement des 2 caractères au cours des années.

II en est de même pour le Tableau III où l'on a relevé l'évolution des caractères de 47 lignées suivies en ligne directe de la $I_{1}$ à $l_{a} I_{5}$, à raison d'une plante autofécondée par descendance chaque année.

Dans les 3 tableaux, l'évolution de la médiane est la même que celle de la moyenne. Pour l'ensemble des lignées, le poids de glomérules récoltés augmente de 2,54 à $9,20 \mathrm{~g}$ (de 150 à 550 glomérules environ) et le pourcentage de glomé- rules viables de 19,44 à 68,41 . Les estimations de la médiane sont généralement inférieures à celle de la moyenne en raison du nombre relativement élevé des poids ou des pourcentages très supérieurs à la moyenne. Cela se traduit par une longue queue de la courbe des fréquences et une disparité entre la moyenne ou la médiane et le centre des étendues.

Le Tableau IV compare, à l'air libre et dans chaque isoloir, le pourcentage de cymes fécondées sur le nombre total de cymes et le pourcentage de glomérules viables sur le nombre de glomérules récoltés pour les 8 racines étudiées. Les résultats sont très voisins. Un test d'appariement après transformation angulaire des données, indique qu'il n'y a pas de différences significatives pour les 2 caractères dans les 2 types d'isolement, ce qui prouve que les conditions de température d'humidité, de lumière et de ventilation à l'intérieur de l'isoloir sont proches de celles qui existent à l'air libre.

Pour l'ensemble des lignées (Tableau I), le pourcentage des descendances comportant à chaque génération au moins $1,3,5,10$ ou 20

Tableau III. Evolution du poids moyen et médian (en $\mathrm{g}$ ) des glomérules récoltés $(\mathrm{g} \mathrm{r})$ et du pourcentage de glomérules viables (\%g v) pour 47 lignées suivies en ligne directe de la $I_{1}$ à la $I_{5}$ (1983-1987).

\begin{tabular}{|c|c|c|c|c|}
\hline GENERATION & & MOYENWE & MEDIANE & ETENDOE \\
\hline $\mathrm{I}_{1}$ & $\begin{array}{l}g r \\
z \mathrm{~g} v\end{array}$ & $\begin{array}{r}5,65 \\
40,00\end{array}$ & $\begin{array}{r}2,50 \\
33,54\end{array}$ & $\begin{array}{c}0,1-45,0 \\
i-100,0\end{array}$ \\
\hline $\mathrm{I}_{2}$ & $\begin{array}{ll}g r \\
z g v\end{array}$ & $\begin{array}{r}8,35 \\
58,40\end{array}$ & $\begin{array}{r}4,91 \\
58,60\end{array}$ & $\begin{array}{c}0,1-39,1 \\
2-100,0\end{array}$ \\
\hline $\mathrm{I}_{3}$ & $\begin{array}{ll}g & r \\
z & g\end{array}$ & $\begin{array}{l}11,16 \\
60,21\end{array}$ & $\begin{array}{r}6,62 \\
63,30\end{array}$ & $\begin{array}{r}0,1-59,7 \\
1-100,0\end{array}$ \\
\hline$I_{4}$ & $\begin{array}{ll}8 & r \\
Z & g\end{array}$ & $\begin{array}{l}12,53 \\
62,45\end{array}$ & $\begin{array}{r}6,90 \\
66,20\end{array}$ & $\begin{array}{r}1-45,5 \\
5-100,0\end{array}$ \\
\hline Is $_{5}$ & $\begin{array}{l}\mathrm{g}_{\mathrm{r}}^{\mathrm{r}} \\
\mathrm{g} \mathrm{v}\end{array}$ & $\begin{array}{l}15,41 \\
74,26\end{array}$ & $\begin{array}{r}9,35 \\
85,35\end{array}$ & $\begin{array}{r}0,8=86,0 \\
10=100,0\end{array}$ \\
\hline
\end{tabular}

Tableau IV. Pourcentage de cymes fécondées et de glomérules viables sous isoloir et à l'air libre (isolements par la distance) pour 8 racines (1986).

\begin{tabular}{|c|c|c|c|c|c|c|c|c|c|c|}
\hline & & 1 & 2 & 3 & 4 & 5 & 6 & 7 & 8 & MorBilit \\
\hline \multirow{2}{*}{$\begin{array}{l}Z \text { DE CYMES } \\
\text { FECONDEES }\end{array}$} & Isoloir & 5,1 & 9,3 & 17,6 & 2,1 & 6,5 & $3, I$ & 13,0 & 0.2 & 7,1 \\
\hline & Air Iibre & 3,0 & 10,2 & 16,2 & 1,5 & 5,7 & 4,1 & 17,6 & 0.6 & 7,4 \\
\hline \multirow{2}{*}{$\begin{array}{c}\text { \% DE GLOMERULES } \\
\text { VIABLES }\end{array}$} & Isoloir & 24 & 22 & 12 & 12 & 24 & 20 & 24 & 18 & 19,5 \\
\hline & Air libre & 26 & 24 & 14 & 13 & 24 & 19 & 26 & 18 & 20,5 \\
\hline
\end{tabular}


plantes pseudocompatibles est indiqué dans le Tableau V. II en ressort que la proportion des lignées ne pouvant s'autoféconder décroit de 6,8 à $1,1 \%$ entre les générations $l_{1}$ et $l_{7}$ et que la proportion des descendances comportant, par exemple, moins de 10 plantes décroît de 34,2 à $28,1 \%$ entre les mêmes générations. II est de plus en plus facile d'obtenir un nombre élevé de plantes dans une descendance à mesure que le nombre des générations d'autofécondation augmente. II persiste cependant, même dans les générations les plus avancées, un nombre important de plantes qui ont de grandes difficultés à s'autoféconder. Ainsi, si on considère qu'un nombre minimal de 5 plantes est nécessaire pour juger une lignée à chaque génération, le Tableau $\checkmark$ montre que $48,3 \%$ seulement des plantes autofécondées en $I_{0}$ peuvent être suivies jusqu'en $I_{4}$. Au niveau d'homozygotie de la $I_{4}$, la fixation des lignées est généralement suffisante. La multiplication des plantes $I_{5}$ à l'air libre, dans un isolement par la distance, ne présente pas de difficultés. Toutes les cymes donnent des glomérules viables en raison d'une pseudocompatibilité élevée et de l'existence, à ce niveau, d'allèles d'incompatibilité différents entre les plantes d'une même génération.

Le Tableau VI donne les pourcentages d'allofécondation pour 60 lignées $I_{1}$ à $I_{7}$ choisies au hasard au cours des 4 années. Ce pourcentage est variable pour chaque génération et il n'y a apparemment pas d'évolution au cours des générations. Les lignées pseudocompatibles, indépendamment de leur degré de consanguinité, ont donc un comportement très différent. Certaines lignées se comportent pratiquement comme des plantes autogames (c'est le cas de 26 des 60 lignées testées). $\mathrm{Ce}$ sont les plus faciles à maintenir et à multiplier. D'autres se comportent comme des lignées presque autostériles (12 des 60 lignées) et il existe tous les types intermédiaires entre ces deux types extrêmes. Des remarques analogues ont déjà été faites sur des plantes $I_{0}$ (Arnold, 1976; Kruse, 1981).

Les 10 descendances d'une plante autofécondée en 1984 ont été suivies au cours des générations $I_{1}$ à $l_{3}$ et comparées pour les caractères, poids de glomérules récoltés et pourcentage de glomérules viables, à l'aide d'un test de Friedmann (Tableau VII). L'analyse des résultats indique que le classement relatif des lignées entre elles n'est pas dû au hasard avec une probabilité inférieure à 0,01 pour le premier caractère et 0,005 pour le second caractère. Les rangs respectifs sont peu modifiés au cours des générations. II existe donc une forte présomption pour l'existence d'un déterminisme génétique de la pseudocompatibilité chez la betterave, déterminisme analogue par exemple à celui décrit chez certains trèfles (Townsend, 1970; Duncan et al., 1973).

Tableau V. Proportion des lignées comportant à chaque génération au moins 1, 3, 5, 10 ou 20 plantes pseudocompatibles (1983-1986).

\begin{tabular}{lccccccc}
\hline \hline & $I_{1}$ & $I_{2}$ & $I_{3}$ & $I_{4}$ & $I_{5}$ & $I_{6}$ & $I_{7}$ \\
\hline I plante au moins & 93,2 & 96,0 & 95,8 & 98,4 & 98,1 & 96,2 & 98,9 \\
3 plantes au moins & 83,7 & 88,7 & 88,1 & 90,6 & 91,2 & 85,4 & 92,2 \\
5 plantes au moins & 78,6 & 83,5 & 83,6 & 88,0 & 88,7 & 81,2 & 86,4 \\
10 plantes au moins & 65,8 & 70,5 & 72,2 & 81,4 & 82,3 & 70,7 & 71,9 \\
20 plantes au moins & 48,4 & 53,2 & 59,7 & 72,0 & 68,5 & 60,5 & 60,9 \\
\hline \hline
\end{tabular}

Tableau VI. Pourcentage d'allofécondation pour 60 lignées pseudocompatibles de betterave fourragère et sucrière en présence de pollen étranger (1983-1986).

\begin{tabular}{lccccccc}
\hline & $I_{1}$ & $I_{2}$ & $I_{3}$ & $I_{4}$ & $I_{5}$ & $I_{6}$ & $I_{7}$ \\
\hline MOMBRE DE LIGREES & 6 & 9 & 10 & 11 & 9 & 7 & 8 \\
\hline Z d'allofécondation & 33,8 & 52,7 & 60,5 & 47,2 & 37,9 & 19,9 & 30,4 \\
Etendue & $0,5-98$ & $3-97$ & $11-100$ & $0-98$ & $0-96$ & $0-77$ & $0-98$ \\
\hline
\end{tabular}


Tableau VII. Classement relatif de 10 lignées de betterave sucrière issues d'une plante $\mathrm{I}_{0}$ pour les caractères poids de glomérules récoltés $(\mathrm{g} r)$ et pourcentage de glomérules viables $\left(\% \mathrm{~g}\right.$ v) au cours des générations $\mathrm{l}_{1}$ à $\mathrm{I}_{3}$ (1985-1987).

\begin{tabular}{|c|c|c|c|c|c|c|c|c|c|c|c|c|}
\hline & & & 1 & 2 & 3 & 4 & 5 & 6 & 7 & 8 & 9 & 10 \\
\hline $\mathrm{I}_{1}$ & 1985 & $\begin{array}{ll}g & r \\
Z & g ~ v\end{array}$ & $\begin{array}{l}5 \\
4\end{array}$ & $\begin{array}{l}3 \\
4\end{array}$ & $\begin{array}{l}6 \\
8\end{array}$ & $\begin{array}{l}4 \\
2\end{array}$ & $\begin{array}{r}10 \\
7\end{array}$ & $\begin{array}{l}9 \\
8\end{array}$ & $\begin{array}{r}8 \\
10\end{array}$ & $\begin{array}{l}1 \\
4\end{array}$ & $\begin{array}{l}7 \\
2\end{array}$ & $\begin{array}{l}1 \\
1\end{array}$ \\
\hline $\mathrm{I}_{2}$ & 1986 & $\begin{array}{ll}\mathbf{g} & \mathbf{T} \\
\mathbf{Z} & \mathbf{g} \mathrm{v}\end{array}$ & $\begin{array}{l}7 \\
5\end{array}$ & $\begin{array}{l}3 \\
2\end{array}$ & $\begin{array}{l}5 \\
1\end{array}$ & $\begin{array}{l}6 \\
9\end{array}$ & $\begin{array}{l}8 \\
7\end{array}$ & $\begin{array}{r}10 \\
7\end{array}$ & $\begin{array}{r}9 \\
10\end{array}$ & $\begin{array}{l}4 \\
2\end{array}$ & $\begin{array}{l}2 \\
6\end{array}$ & $\begin{array}{l}1 \\
2\end{array}$ \\
\hline $\mathbf{I}_{\mathbf{3}}$ & 1987 & $\begin{array}{l}g \mathrm{r} \\
Z_{\mathrm{g}} \mathrm{g} \mathrm{v}\end{array}$ & $\begin{array}{l}8 \\
5\end{array}$ & $\begin{array}{l}2 \\
4\end{array}$ & $\begin{array}{l}7 \\
1\end{array}$ & $\begin{array}{l}4 \\
8\end{array}$ & $\begin{array}{l}5 \\
7\end{array}$ & $\begin{array}{r}10 \\
9\end{array}$ & $\begin{array}{r}9 \\
10\end{array}$ & $\begin{array}{l}3 \\
3\end{array}$ & $\begin{array}{l}6 \\
5\end{array}$ & $\begin{array}{l}1 \\
1\end{array}$ \\
\hline
\end{tabular}

\section{DISCUSSION}

Les équations des droites de régression entre les valeurs moyennes et médianes des deux caractères pour l'ensemble des lignées (Tableau I) et le coefficient de consanguinité des générations $I_{1}$ à $I_{7}$ ont été calculés. Les coefficients de régression sont tous très significatifs. Les coefficients de détermination entre les valeurs observées et calculées sont supérieurs à $0,92(5 \mathrm{ddl})$. II y a donc une relation linéaire très étroite entre la croissance des 2 variables et le coefficient de consanguinité ce qui confirme le caractère génétique de la pseudocompatibilité. Les 2 caractères évoluent parallèlement (les coefficients de détermination sont supérieurs à 0,84 ). En particulier, plus on récolte de glomérules, plus ces glomérules ont une bonne faculté germinative.

Le passage de la génération $I_{1}$ à la génération $I_{7}$ multiplie par 3,6 la valeur médiane du poids des glomérules récoltés et par 3,5 la valeur médiane du pourcentage des glomérules viables pour l'ensemble des lignées (Tableau I). Une progression encore plus importante est observée si on suit l'évolution de ces caractères pour les lignées $I_{1}$ de 1983 jusqu'aux lignées $I_{4}$ de 1986 qui en sont issues (Tableau II). La même évolution se retrouve dans le tableau III. L'autofécondation répétée des lignées constitue donc un moyen efficace de sélectionner des génotypes de plus en plus pseudocompatibles.

Cette évolution, confirmée également par les travaux des auteurs soviétiques cités ci-dessus en introduction, est, par contre, en contradiction avec les conclusions de Larsen $(1975,1977 \mathrm{~b}$, 1983). Ce dernier constate, en effet, que la pseudocompatibilité est une fonction exponentielle du degré d'hétorozygotie pour les 4 loci des séries alléliques $\mathrm{S}$. II en déduit que plus une plante est hétérozygote, plus elle devient pseudocompatible. Il en résulterait une réduction de la pseudocompatibilité avec l'augmentation de degré d'in- breeding et, à la limite, une valeur nulle pour une plante homozygote. Selon cet auteur, plus une plante est hétérozygote, plus elle serait susceptible de synthétiser une substance diminuant l'aptitude du style à empêcher la fécondation par du pollen incompatible. Cette substance serait synthétisée à une température élevée, voisine de $35^{\circ} \mathrm{C}$ (Larsen, 1984), température à laquelle il est recommandé de sélectionner les lignées pseudocompatibles. Ces expériences ont été réalisées en serre au cours des étés très chauds de 1975 et 1976 en utilisant comme isoloirs des sacs en papier-parchemin de $6 \times 22 \mathrm{~cm}$. De telles conditions sont très différentes des nôtres : expérimentation en plein champ avec des isoloirs dont on a vérifié au préalable que le climat interne est voisin de celui existant à l'air libre.

Nos résultats font apparaître une corrélation inverse entre la force de l'incompatibilité et le niveau d'homozygotie du style. A la limite, l'importance de la pseudocompatibilité pourrait être un indicateur du degré de consanguinité et de l'homozygotie des séries alléliques. Cette situation est en opposition avec la théorie de l'autoincompatibilité chez la betterave (Larsen, 1977a) qui considère que l'incompatibilité n'existe qu'en cas d'identité des séries alléliques dans le pollen et le style. Cela ne peut apparemment s'expliquer qu'en admettant que le système génétique conditionnant la pseudocompatibilité est distinct de celui conditionnant l'incompatibilité gamétophytique. D'une manière analogue aux observations de Dana \& Ascher sur Petunia integrifolia, la pseudocompatibilité chez la betterave apparaît comme un effet de la sélection et elle est partiellement héritable.

Un recensement général des lignées $I_{0}$ suivies jusqu'en $\mathrm{I}_{4}$ au cours des 10 dernières années, indique que, sur un total de 971 plantes $I_{0}$ autofécondées durant cette période dans les familles autostériles, 452 descendances, soit $46,5 \%$ avaient présenté un nombre de plantes suffisant 
durant 4 générations successives. Ce pourcentage est voisin de $48,3 \%$, déterminé théoriquement ci-dessus à partir d'un effectif minimum de 5 plantes par génération. II est donc possible, sous nos conditions, d'obtenir en moyenne, une lignée fixée pseudocompatible à partir de 2 plantes autofécondées dans une population de base d'origine. II serait intéressant de pouvoir comparer ce bilan au bilan de la méthode de diploïdisation des lignées obtenues par gynogénèse, méthode qui fait actuellement l'objet de recherches intensives dans plusieurs établissements de sélection betteravière.

Si on constate une homogénéisation rapide de l'aspect des lignées dès la première ou deuxième génération d'autofécondation, ce n'est en pratique qu'à partir de la quatrième ou cinquième génération que l'on atteint un taux de pseudocompatibilité suffisant pour pouvoir juger la valeur propre des lignées et tester leur aptitude à la combinaison.

II faut par ailleurs être averti, quelles que soient les précautions prises, du danger toujours présent des hybridations accidentelles dues à l'allopollen. Un grand nombre de lignées s'autofécondent mal et acceptent très facilement le pollen étranger. La persistance d'un tel pollen à l'intérieur de l'isoloir a été décelée en isolant en 1985 et 1988, 14 plantes mâle-stériles choisies à l'intérieur d'un champ de porte-graines. Le nombre de cymes fécondées viables par isoloir a été en moyenne de $1,1 \%$ o du nombre total des cymes isolées (de 0 à $3,9 \%$ ). II existe donc relativement peu de fleurs qui puissent être fécondées par le pollen emprisonné à l'intérieur de l'isoloir. Ce nombre n'est cependant pas négligeable si on le rapporte au nombre relatif des cymes autofécondées viables obtenues au cours des premières générations en particulier, et qui est par exemple de $6,2 \%$ pour la valeur médiane de la $I_{1}$ (Tableau I). Le risque est grand à ce niveau, où il existe de nombreuses lignées à faible taux de pseudocompatibilité, d'être amené à autoféconder des plantes hybrides (le gène marqueur $R$ de coloration rouge de l'hypocotyle ne permet pas de les reconnaître toutes, l'année suivante). $\mathrm{Ce}$ risque se renouvelle à chaque génération. II nécessite donc une grande attention et constitue en fait, la principale difficulté que nous avons rencontrée dans la sélection de lignées pseudocompatibles par autofécondation.

L'obtention de lignées homozygotes de betterave, si elle est parfaitement possible dans des conditions de milieu bien définies, ne devrait cependant pas être considérée comme un objectif en soi. L'efficacité du travail d'isolement et de sélection de bonnes lignées fixées est en effet d'abord conditionnée par la recherche préalable de populations de base de valeur propre élevée et possédant une très bonne aptitude à la combinaison.

\section{RÉFÉRENCES}

Archimowitsch A. (1948) Control of pollination in sugar beet. Bot. Rev., 15, 613-628

Arnold M.H. (1976) Annual report. Plant Breed. Inst. Cambridge 84-85

Bandlow G. (1965) Untersuchungen über Inzuchtlinien der Zuckerrübe und ihre Kombinationseignung. Züchter 35, 6, 239-250

Brewbaker H.E. (1934) Self fertilization in sugar beets as influenced by type of isolators and other factors. J. Agric. Res. 48, 323-337

Codrescu V. (1974) Effet de la sélection et de l'hybridation des lignées consanguines pseudo-auto-incompatibles chez la betterave à sucre. Z. Pflanzenzucht. $72,9-25$

Coons G.H., Owen F.V. \& Stewart D. (1955) Improvement of the sugar beet in the United States. Adv. Agron. 7, 89-139

Dana M.N. \& Ascher P.D. (1985) Pseudo-self-compatibility (PSC) in Petunia integrifolia. J. Hered. 76, 468470

Dobrosotskov V.V. (1979) Results of work on the production of sugar beet lines. Plant Breed. Abst. 5327, 1980

Duncan J.F., Anderson M.K. \& Taylor N.L. (1973) Effect of inbreeding on pseudoself-compatibility in red clover (Trifolium pratense L.). Euphytica 22, 535-542

Grinko T.F. (1930) Self pollination in the sugar beet. Proc. USSR Congr. Genet. Pl. An. Breed. 4, 111-119

Henny R.J. \& Ascher P.D. (1976) The inheritance of Pseudo-Self-Compatibility (PSC) in Nemesia strumosa Benth. Theor. Appl. Genet. 48, 185-195

Hilleshög (1974) Brochure de présentation des Etablissements Hilleshög Frö AB. P.O. Box 302, S. 26123 Landskrona, $37 \mathrm{p}$

Kohls H.L. \& Down E.E. (1934) Influence of inbreeding and selection on seed production of space-isolated mother beets. J. Am. Soc. Agron. 26, 327-332

Kruse A. (1981) The potential use of heterosis in Beta vulgaris L. II. Yield performance of pure lines and their hybrids. Euphytica 30, 791-802

Larsen K. (1975) Correlation between the quadruple heterozygous $S$. genotype $S_{a}^{1-2}-S_{b}^{3 \cdot 4}-S_{c}^{5 \cdot 6}$ $\mathrm{S}_{\mathrm{d}}^{7-8}$ and pseudocompatibility in Beta vulgaris $\mathrm{L}$. Incompatibility Newsletter 5, 85-88

Larsen K. (1977a) Self incompatibility in Beta vulgaris L. I. Four gametophytic complementary S-loci in sugar beet. Hereditas $85,227-248$

Larsen K. (1977b) Pseudocompatibility in Beta vulgaris L. A quantitative character, dependent on the degree of S-gene heterozygosity. Incompatibility Newsletter 8, 48-51

Larsen K. (1983) Incompatibility pseudo-compatibility and preferential fertilization in Beta vulgaris L. In : Pollen : Biology and implications for plant breeding. (D.L.. Mulcahy and E. Ottaviano eds.) Elsevier New York, 205-210 
Larsen K. (1984) S-heterozygosity-dependent pseudocompatibility in Beta vulgaris L. : response to temperature. Plant cell. Incompatibility Newsletter 16, 4-6

Le Cochec F. (1982) Les variétés monogermes de betterave sucrière. Suggestions pour la sélection d'un autre type de variétés: les hybrides $F_{1}$ ou hybrides entre deux lignées fixées. Sélectionneur Français 30, $45-48$

Longden P. C., Johnson M.G. \& Love B. (1971) Sugar beet seedling emergence prediction from radiographs. J. Int. Inst. Sugar Beet. Res. 5, 160-168

Lynes F.F. \& Cormany C.E. (1942) Refinements in the technique of Isolating by bags and cages. Proc. Am. Soc. Sugar Beet Technol. 3, 399-405

Maletsky S.I. \& Weisman N.J. (1978) A population Genetic Analysis of Self and Cross-Incompatibility in Sugar Beet (Beta vulgaris L.) Theor. Appl. Genet. 52, 21-28

Nilsson H. (1923) Praktische Rübenzüchtung in Svälof nach neuen Methoden. Sver. Utsädes foren. Tidskr. $33,75-92$

Oldemeyer R.K. (1986) Communication personnelle, 16 juillet. Mono-Hy. Longmont. Colorado 80501, 3 pp Oldemeyer R.K. \& Smith P.B. (1965) Les conséquences de l'application de la méthode d'hybridation dans la sélection de la betterave sucrière. Communication $n^{\circ}$ 13. $28^{\mathrm{e}}$ Congrès d'Hiver. Inst. Intern. Rech. Betterav. Bruxelles. $14 \mathrm{pp}$
Owen F.V. (1942) Inheritance of cross and self sterility in Beta vulgaris L. Agric. Res. 64, 679-698

Savitsky H. (1950) A method of determining self fertility and self sterility in sugar beets, based upon the stage of ovule development shortly after flowering. Proc. Am. Soc. Sugar Beet Technol. 6, 198-201

Scott R.K. (1970) The effect of weather on the concentration of pollen within sugar beet crops. Ann. Appl. Biol. 66, 119-127

Shaw H.B. (1916) Self close and cross-fertilization of beets. Mem. New York Bot. Garden 6, 149-152

Stewart G. (1933) Some effects of inbreeding in sugar beets. J. Am. Soc. Agron. 25, 4, 237-258

Townsend C.E. (1970) Inheritance of a self-compatibility response to temperature and the segregation of $S$ alleles in diploid alsike clover, Trifolium hybridum $\mathrm{L}$. Crop. Sci. 10, 558-562

Vashchenko T.G. \& Yusubov A.M. (1986) Producing new inbred lines of beet. Plant Breeding Abstracts 10 993,1987

Vilmorin (De) J. (1923) L'hérédité chez la betterave cultivée. Gauthier-Villars, Paris, 153 pp

Weisman N.J. (1981) Pseudocompatibility (PSC) in Beta vulgaris $L$. populations : dependance of the level of inbreeding and temperature. Incompatibility Newsletter $13,138-140$ 